\title{
Trust within your team during a pandemic
}

Elizabeth Sturgiss, Alexander M Clark

TRUST is the basis for all high-functioning teams. General practitioners (GPs) work as part of diverse teams - clinical practice teams, policy and governance teams, teaching and education teams, and research teams. All of these roles are an important part of being a GP and reflect the truly generalist specialty of general practice. ${ }^{1}$ The concept of trust has been an intense focus within the business setting, ${ }^{2}$ and general practice teams can learn from this during these challenging times.

One useful framework of 'trust in the workplace ${ }^{3}$ recognises four essential components:

1. Reliability - team members doing what they say they will do

2. Sincerity - meaning what you say, and saying what you mean

3. Competence - doing your best work while also seeking help when you need it, and being willing to learn from others

4. Care-having genuine regard for the wellbeing of the other members of your team. ${ }^{3}$

We are already seeing general practice teams successfully adapting their daily work and continuing to provide high-quality care. Practices are looking after their staff who are at risk of COVID-19 by adapting their working conditions and protecting them from infection. I have seen exceptional peer support across networks, with GPs using social media and messaging services to provide support for each other. I am impressed by the strong advocacy from The Royal Australian College of General Practitioners and other representative organisations for safe workplace conditions for clinicians and other general practice staff.
However, not all GPs are working in teams that are giving them a sense of comfort and control. The above trust framework can direct reflection if you are coping in a team that is not performing as well as it needs to be. Framing your feedback around one of the four components may help you communicate the changes that you need in your team for optimal functioning.

For example, are you feeling that your team genuinely cares about your wellbeing and workplace safety? Are there reliable systems in place that reduce the risk to your team? Are your colleagues doing what they say they will do? Sometimes in a pressured environment people may feel they have to say yes to more things - but, as always, when people give a 'fuzzy' yes it is much worse than a firm no.

During the coming months, our ability to provide high-quality patient care, contribute to evolving policy and maintain our excellence in teaching and research will depend on our ability to build and maintain trust within high-functioning teams.

First published online 6 April 2020.

\section{Authors}

Elizabeth Sturgiss PhD, FRACGP, BMed, MPH, MForensMed, Senior Lecturer, Department of General Practice, Monash University, Vic. liz.sturgiss@monash.edu

Alexander M Clark PhD, FCAHS, Vice President Research \& Innovation Office, University of Alberta, EDM

Competing interests: None.

Provenance and peer review: Commissioned, peer reviewed.

Citation: Sturgiss E, Clark AM. Trust within your team during a pandemic. Aust J Gen Pract 2020;49 Suppl 5. doi: 10.31128/AJGP-COVID-05.

\section{References}

1. Reeve J. Primary care redesign for person-centred care: Delivering an international generalist revolution. Aust J Prim Health 2018;24(4);330-36. doi: 10.1071/PY18019.
2. Zak PJ. The Neuroscience of Trust. Boston, MA: Harvard Business Review, 2020. Available at https://hbr.org/2017/01/the-neuroscience-of-trust [Accessed 28 March 2020].

3. Feltman C. The thin book of trust: An essential primer for building trust at work. Bend, OR: Thin Book Publishing, 2009. 